\section{Consideraciones en redacción científica: el título, resumen y palabras clave}

\section{Scientific writing considerations: the title, abstract and keywords}

\section{Resumen}

Con el término redacción científica se incluye a la totalidad de normas y protocolos estandarizados para lograr la adecuada escritura y difusión de manuscritos en revistas científicas. Los primeros segmentos de un artículo científico suelen ser los más leídos y de los que depende que el lector se anime por leer la totalidad del mismo. La apropiada redacción de un título, resumen y palabras clave permite que el lector comprenda y se interese por la investigación realizada. De la misma forma, simplifica su búsqueda en bases de datos y mejora su difusión con mayor rapidez. En la presente nota científica se resumen las principales recomendaciones y guías para redactar apropiadamente un título, un resumen y las palabras clave de un artículo científico.

Palabras clave: Comunicación y divulgación científica; Escritura médica; Odontología; Publicaciones de divulgación científica.

\begin{abstract}
The term scientific writing includes all standardized norms and protocols to achieve the adequate writing and diffusion of manuscripts in scientific journals. A scientific article's first segments are usually the most read and depending on them the reader is encouraged to read the entire article. The proper wording of a title, abstract and key words allows the reader to understand and be interested in the research carried out. In addition, it simplifies the article's search in databases and improves to more quickly diffusion. This scientific note summarizes the main recommendations and guidelines for the proper way to write a scientific article`s title, abstract and key words.
\end{abstract}

Keywords: Dentistry; Medical writing; Publications for science diffusion; Scientific communication and diffusion.

\section{Nota Científica}

Yuri Castro-Rodríguez ${ }^{1, a}$, Manuel Antonio Mattos-Vela ${ }^{1, b}$, Aron Aliaga-Del Castillo ${ }^{2, c}$

${ }^{1}$ Facultad de Odontología. Universidad Nacional Mayor de San Marcos. Lima, Perú.

2 Facultad de Odontología de Bauru. Universidad de São Paulo. São Paulo, Brasil.

a Magíster en Educación

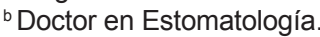

${ }^{c}$ Maestro en Ortodoncia.

Correspondencia:

Yuri Castro-Rodríguez

Correo electrónico: yuricastro_16@hotmail.com Jr. Tomás Catari 463, Urb. El Trébol. Dpto. 201. Lima 39, Perú

\section{Coautores: \\ Manuel Antonio Mattos-Vela mmattosv@unmsm.edu.pe \\ Aron Aliaga-Del Castillo \\ a_aliaga@hotmail.com}

Conflicto de intereses: Los autores declaran no tener conflictos de interés.

Fuente de financiamiento: Autofinanciado

Fecha de recepción: 18/01/18

Fecha de aceptación: 27/02/18 


\section{Introducción}

La producción y publicación de textos científicos permite informar los resultados disciplinares útiles para una sociedad y comunidad científica. Producir textos científicos implica el conocimiento de reglas y convenciones que se engloban dentro de la redacción científica. Los artículos científicos se caracterizan por ser claros, precisos, con un lenguaje planificado, formal, uso de términos específicos y basándose en estructuras protocolizadas ${ }^{1,2}$, características esenciales que los editores y revisores (árbitros) consideran al momento de evaluar un manuscrito para su posible publicación.

La redacción científica permite a los investigadores representar en las letras el mundo que están investigando según cómo ellos lo comprenden y experimentan. Es común encontrar que los manuscritos que son enviados a revistas científicas son rechazados y no valorados principalmente por la incorrecta redacción que utilizó el investigador, esto debido a que suponemos que como investigadores "sabemos escribir". Es menester que el investigador y equipo que desarrolló la investigación se capacite y demuestre habilidades en la redacción científica como una estrategia comunicativa y aporte a la transferencia del conocimiento ${ }^{1,3}$. Un buen investigador también debe ser un buen comunicador pues una mala redacción puede impedir o dificultar la publicación de un manuscrito con potencial ${ }^{2}$.
En muchas ocasiones, de la totalidad del artículo científico, los editores y lectores solo leerán el título y resumen del documento; dependiendo del interés que se generó en el lector se continuará con los siguientes segmentos. Lamentablemente, la mayoría de errores en redacción científica se encuentran en los títulos (principalmente mala sintaxis) y en el resumen (falta de claridad, ausencia de precisión y falta de información básica importante). Por tal motivo, es necesario valorar la redacción científica de los primeros segmentos como el título y resumen para atraer al lector y que logre ser recuperada en las bases de datos. En la presente nota científica se resumen los principales aspectos a considerar en la redacción de un artículo científico (título, resumen y palabras clave).

\section{El título}

Es el primer elemento de un artículo científico. Permite identificar la obra y por lo general el lector sabrá cuál es el objeto de estudio y decidirá si es de su interés ${ }^{4}$. A nivel académico-científico el título se presenta como una frase coherente y completa que otorga las nociones para entender el enfoque del tema y ser el hilo conductor (Tabla 1).

El título es la carta de presentación hacia el lector ${ }^{3}$. Algunos autores cometen ciertos errores que limitan su entendimiento y su rápida ubicación en bases de datos dejando de llamar la atención del lector (Tabla 2).

Tabla 1. Consideraciones a resaltar al momento de redactar un título

\begin{tabular}{|c|c|}
\hline Características & Recomendaciones para su redacción \\
\hline $\begin{array}{l}\text {-Preciso, breve y completo, sin sobrecargarlo con } \\
\text { elementos indicativos. } \\
\text {-Utilizar el menor número de palabras, sin caer en } \\
\text { extremos }{ }^{4,5} \text {. } \\
\text {-Se sugiere una extensión entre } 10-15 \text { palabras } \\
\text { (incluido preposiciones, artículos y conjunciones) }{ }^{6} \text {. } \\
\text {-Carece de abreviaciones. } \\
\text {-Debe motivar la lectura del artículo, es la imagen y } \\
\text { etiqueta de la investigación a publicar }{ }^{7} \text {. } \\
\text {-La sintaxis (orden de las palabras) debe ser } \\
\text { adecuada. } \\
\text {-Precaución con las concordancias y requerimientos } \\
\text { gramaticales. }\end{array}$ & $\begin{array}{l}\text {-Con una lluvia de ideas intentar buscar palabras o ideas imprescindibles. } \\
\text {-No es necesario que sea igual al título del proyecto de investigación. } \\
\text {-Evitar que se asemeje a un relato de enunciados; no necesariamente posee la estructura de } \\
\text { sujeto y predicado. } \\
\text {-Títulos inapropiados no serán localizados y no llegarán al público de destino. } \\
\text {-Comenzar con títulos provisionales, luego mejorarlo poco a poco. } \\
\text {-Evitar la redacción de fórmulas, abreviaturas, jergas, caracteres poco conocidos o términos de } \\
\text { difícil pronunciación. } \\
\text {-Preguntarnos cómo lo encontraríamos en una base de datos. } \\
\text {-Precaución con el uso de gerundios pues conllevan a la confusión. } \\
\text {-Evitar los títulos en serie como: Primera parte, segunda parte. Cada artículo debe ser } \\
\text { independiente y coherente (excepto en los casos de ser requerido por la revista, en forma de } \\
\text { invitación para publicar) }\end{array}$ \\
\hline
\end{tabular}

Tabla 2. Ejemplos de errores frecuentes al momento de redactar títulos

\begin{tabular}{|c|c|c|c|}
\hline Errores frecuentes & Ejemplo & Consideración & Sugerencia de cambio \\
\hline Títulos ambiguos. & $\begin{array}{l}\text { Cambios dimensionales del } \\
\text { alginato, una evaluación del } \\
\text { tiempo y almacenajes. }\end{array}$ & $\begin{array}{l}\text { No es comprensible si se realizó la evaluación del tiempo, } \\
\text { del almacenaje o de los cambios dimensionales. }\end{array}$ & $\begin{array}{l}\text { Cambios dimensionales del alginato } \\
\text { según variación del tiempo y } \\
\text { condiciones de almacenaje. }\end{array}$ \\
\hline Redactar acciones. & $\begin{array}{l}\text { Efecto del trauma oclusal } \\
\text { sobre las recesiones } \\
\text { gingivales. }\end{array}$ & $\begin{array}{l}\text { En lo posible evitar las acciones metodológicas como: } \\
\text { "Efecto", "Análisis", "Evaluación", etc. Que deben ser } \\
\text { explicadas en la sección de material y métodos.* }\end{array}$ & $\begin{array}{l}\text { Trauma oclusal primario y su relación } \\
\text { con la severidad de las recesiones } \\
\text { gingivales. }\end{array}$ \\
\hline $\begin{array}{l}\text { Uso de términos } \\
\text { redundantes y/o } \\
\text { palabras que no } \\
\text { aportan. }\end{array}$ & $\begin{array}{l}\text { Midazolam y su accionar en } \\
\text { la sedación consciente. Un } \\
\text { estudio experimental. }\end{array}$ & $\begin{array}{l}\text { Los términos metodológicos y resultados deben evitarse } \\
\text { en el título; suelen ser redundancias: "Una investigación } \\
\text { experimental de...", "Un estudio de..." "Contribución } \\
\text { a..." "Aspectos interesantes sobre..." "Informe } \\
\text { preliminar..." "Observaciones sobre". }\end{array}$ & $\begin{array}{l}\text { Midazolam como alternativa de } \\
\text { tratamiento odontopediátrico en la } \\
\text { sedación consciente. Ensayo clínico } \\
\text { aleatorizado. }\end{array}$ \\
\hline $\begin{array}{l}\text { Demasiado extenso/ } \\
\text { breve }\end{array}$ & $\begin{array}{l}\text { Rehabilitación oral } \\
\text { multidisciplinaria de un } \\
\text { paciente con agenesia múltiple } \\
\text { de incisivos superiores, un } \\
\text { relato de caso clínico. } \\
\text { El periodontograma. }\end{array}$ & $\begin{array}{l}\text { Evitar títulos largos, altisonantes como pequeños } \\
\text { resúmenes y a veces incomprensibles. Por el contrario, } \\
\text { títulos demasiado cortos no resultan atractivos para el } \\
\text { lector pues no indican el tema central del artículo. }\end{array}$ & $\begin{array}{l}\text { Abordaje multidisciplinario de la } \\
\text { agenesia de incisivos superiores. } \\
\text { Informe de caso. } \\
\text { El periodontograma: una herramienta } \\
\text { para el diagnóstico y tratamiento } \\
\text { periodontal. }\end{array}$ \\
\hline
\end{tabular}

*Nota: La pauta CONSORT ${ }^{8}$ recomienda que para los estudios ensayos clínicos se redacte en el título las palabras: "Ensayo clínico aleatorizado". La pauta PRISMA9 recomienda que para revisiones sistemáticas se redacte en el título las palabras: "Revisión sistemática", "Metanálisis" o ambos. Las pautas STROBE ${ }^{10}$ y STARD ${ }^{11}$ recomiendan redactar en el título o en el resumen el diseño de estudio. La pauta SPIRIT recomienda que para protocolos de ensayos clínicos se especifique en el título los siguientes puntos: diseño, población, intervención y en lo posible la sigla o abreviatura del ensayo ${ }^{12}$ La pauta CARE recomienda que para casos clínicos se redacte en el título los términos "Informe de Caso" 13 . 
El título puede ser redactado antes de iniciar el trabajo, en el transcurso de la redacción o al finalizar el artículo ${ }^{3}$; es un tema de elección, lo recomendable es ensayar con varios títulos e ir modificándolo de acuerdo a las apreciaciones de los integrantes del equipo de investigación durante todo el proceso de investigación. Exige dedicarle el tiempo suficiente para su elaboración pues deberá ser auto explicativo y llamar la atención del lector. La comunidad científica suele seleccionar sus lecturas de interés acorde a los títulos y resúmenes. Adicionalmente, el éxito y efectividad de los servicios de indización dependen de la precisión de los títulos ${ }^{3}$. Comúnmente, los títulos inapropiados pueden perderse en la red y no ser recuperados por los lectores, limitando su capacidad de difusión y citación.

\section{El resumen}

Segmento que sintetiza la investigación realizada, también es denominado sumario, extracto, compendio o sinopsis ${ }^{3}$. Cada certamen y revista científica suele tener protocolos diferentes sobre su extensión, redacción y estructura; esto debido a que presenta variaciones según su finalidad y el contexto en el cual se publica ${ }^{1}$. Dependiendo de su clasificación existen ciertas características a considerar durante su redacción (Tabla 3).

El resumen es la primera información que revisa el editor y los revisores, después del título. En la mayoría de veces, la decisión de rechazo y/o aceptación del artículo dependerá de la redacción del resumen. Al lector le permite reconocer el contenido y es parte fundamental para el registro en bibliotecas y bases de datos de investigación ${ }^{1}$. Durante su redacción se debe seguir al objetivo planteado, pensar en los términos que al lector le interesan así como en las expresiones más sencillas para que el público y los editores puedan acceder al artículo una vez publicado. La extensión y estructura del resumen, pueden variar dependiendo del tipo de artículo ${ }^{17-19}$ (Tabla 4).

Tabla 3. Consideraciones para la redacción del resumen

\begin{tabular}{|c|c|c|c|}
\hline \multirow{2}{*}{ Características } & \multirow{2}{*}{ Recomendaciones para su redacción } & \multicolumn{2}{|c|}{ Clasificación } \\
\hline & & Estructurado & No estructurado \\
\hline $\begin{array}{l}\text {-Sus segmentos deben otorgar la idea clara } \\
\text { y exacta del tema a tratar en el artículo. } \\
\text {-Carece de citaciones, tablas y/o figuras. } \\
\text {-Se redacta en tiempo pasado (pretérito). } \\
\text {-Debe despertar el interés del lector para } \\
\text { continuar con los siguientes segmentos. } \\
\text {-Debe ser auto explicativo ya que puede } \\
\text { sustituir al documento }{ }^{14} \text {. } \\
\text {-Según el tipo de artículo y las exigencias } \\
\text { de las revistas su extensión oscila entre } \\
\text { 100-300 palabras } 2,15 \text {. } \\
\text {-En español e inglés (abstract) deben } \\
\text { poseer el mismo contenido. } \\
\text {-Se redacta en un solo párrafo aunque } \\
\text { algunas revistas sugieren redactar según } \\
\text { segmentos. Un buen resumen va seguido } \\
\text { de un buen artículo }{ }^{5} \text {. }\end{array}$ & $\begin{array}{l}\text {-Es la presentación abreviada de la investigación, } \\
\text { de preferencia evitar opiniones personales e } \\
\text { interpretaciones. } \\
\text {-De preferencia no utilizar siglas o abreviaturas excepto } \\
\text { las de dominio general. } \\
\text {-Evitar el uso innecesario de verbos así como presentar } \\
\text { resultados secundarios y hacer inferencias con } \\
\text { resultados no concluyentes }{ }^{3} \text {. } \\
\text {-Evitar expresiones en otro idioma, ejemplos y } \\
\text { enunciados negativos. } \\
\text {-Reflejar la estructura del artículo a presentar. } \\
\text {-Debe ser autosuficiente e inteligible. } \\
\text {-La conclusión debe guardar relación con la del artículo. } \\
\text {-Evitar la primera persona y preferir la redacción en } \\
\text { tercera persona o utilizar el impersonal }{ }^{1} \text {. } \\
\text {-Redactarlo al finalizar la investigación }{ }^{15} \text {. } \\
\text {-Considerar que probablemente será el único segmento } \\
\text { que lea el editor y/o lector. Por tal motivo, deberá causar } \\
\text { la mejor impresión. }\end{array}$ & $\begin{array}{l}\text {-También denominado } \\
\text { informativo, } \\
\text { comprensivo, analítico } \\
\text { o secuencial. } \\
\text {-Al lector le permite } \\
\text { identificar diferentes } \\
\text { elementos (o secciones) } \\
\text { del estudio. } \\
\text {-Es el recomendado } \\
\text { para artículos } \\
\text { originales y revisiones } \\
\text { sistemáticas }{ }^{16} \text {. } \\
\text {-Su formato es variado } \\
\text { pero comúnmente } \\
\text { incluye: Objetivo, } \\
\text { Material y métodos, } \\
\text { Resultados y } \\
\text { Conclusión. }\end{array}$ & $\begin{array}{l}\text {-Denominado indicativo } \\
\text { o descriptivo. } \\
\text {-Precisan el tema del } \\
\text { artículo y el objetivo }{ }^{1} \text {. } \\
\text {-Propio de ensayos, } \\
\text { reportes de caso, } \\
\text { artículos de revisión, } \\
\text { artículos metodológicos, } \\
\text { etc. } \\
\text {-Poseen un formato } \\
\text { diferente según el tipo } \\
\text { de artículo }{ }^{1} \text {. }\end{array}$ \\
\hline
\end{tabular}

Tabla 4. Principales estructuras del resumen de los artículos científicos

\begin{tabular}{|c|c|c|}
\hline Artículo original & Reporte de caso & Artículo de revisión \\
\hline $\begin{array}{l}\text { Las investigaciones primarias y las revisiones } \\
\text { sistemáticas presentan como estructura: } \\
\text { Antecedentes: * El resumen debe proporcionar el } \\
\text { contexto o antecedentes del estudio y declarar el } \\
\text { propósito del mismo }{ }^{21} \text {. } \\
\text { Métodos: Incluir el diseño metodológico, muestra } \\
\text { de estudio, procedimientos o métodos utilizados, } \\
\text { principales variables evaluadas y los métodos } \\
\text { estadísticos. } \\
\text { Resultados: Resumir los datos recolectados más } \\
\text { relevantes, combinar datos descriptivos con } \\
\text { inferenciales. } \\
\text { Conclusiones: Interpretación general de los } \\
\text { resultados del estudio e implicaciones importantes. }\end{array}$ & $\begin{array}{l}\text { El resumen para un artículo de estudio de caso } \\
\text { clínico incluye }{ }^{13} \text { : } \\
\text { Antecedentes: Aporte nuevo del caso. } \\
\text { Descripción del caso: Redactar las principales } \\
\text { características del sujeto, de la naturaleza del } \\
\text { problema (patología), principales signos y } \\
\text { síntomas, tratamiento realizado y resultados } \\
\text { clínicos, radiográficos y/o histológicos. } \\
\text { Conclusión: Principales lecciones que se } \\
\text { pudieron aprender del caso. } \\
\text { A diferencia de los artículos originales su } \\
\text { extensión puede ser entre } 100-200 \text { palabras. }\end{array}$ & $\begin{array}{l}\text { Para las revisiones de tema la estructura } \\
\text { suele poseer: El tema en una oración, } \\
\text { objetivo o tesis, las fuentes consultadas (si } \\
\text { fuera necesario) y la conclusión. } \\
\text { Para los estudios metodológicos su } \\
\text { estructura posee: Tipo de método a } \\
\text { proponer, se evalúa o discute, principales } \\
\text { características del método, aplicación del } \\
\text { método, comportamiento del método y la } \\
\text { conclusión. } \\
\text { A diferencia de los artículos originales su } \\
\text { extensión puede ser entre } 150-200 \text { palabras. }\end{array}$ \\
\hline
\end{tabular}


En los resúmenes estructurados se recomienda entre 2-5 líneas por segmento, dependiendo de las exigencias de las revistas (Tabla 5).

El resumen de las revisiones sistemáticas requiere una especial consideración puesto que debe incluir: Objetivos, pregunta de investigación que incluya componentes como participantes, intervenciones y comparaciones. Métodos, mencionar los criterios de elegibilidad, fuentes de información, bases de datos, fechas de búsqueda, y métodos para evaluación del riesgo de sesgo. Resultados, estudios incluidos y características relevantes, principales resultados (si se realiza metanálisis, incluir medidas de resumen con sus respectivos intervalos de confianza), descripción de los efectos encontrados (mencionado si hubo algún grupo favorecido). Discusión y Conclusiones, breve resumen de las fortalezas y limitaciones de la evidencia y la interpretación general de los resultados e implicaciones clínicas. Otros, en este punto se men- cionan las fuentes de financiamiento de la revisión y el registro del protocolo ${ }^{20}$.

Para ensayos clínicos aleatorizados, la guía CONSORT for abstracts ${ }^{21}$ recomienda incluir: Diseño del ensayo clínico aleatorizado. Método, debe incluir a los participantes, intervenciones, objetivos, definición de los resultados primarios, aleatorización, cegamiento. Resultados, número de participantes aleatorizados en cada grupo, reclutamiento, número de participantes analizados en cada grupo, resultados de cada grupo y el tamańo de efecto estimado, así como su precisión y efectos adversos importantes. Conclusiones, interpretación general de los resultados. Otros, nombre y número de registro del ensayo, y fuente de financiamiento si tuviese.

Para estudios observacionales se recomienda incluir: Antecedentes, objetivo, diseño, contexto, pacientes, mediciones, resultados, limitaciones y conclusiones ${ }^{10}$.

Tabla 5. Ejemplos de resúmenes de los artículos científicos más comunes

\begin{tabular}{|c|c|c|}
\hline Artículo original & Reporte de caso & Artículo de revisión \\
\hline $\begin{array}{l}\text { Título: Factores que contribuyen en la } \\
\text { producción científica estudiantil. El caso de } \\
\text { Odontología. }\end{array}$ & $\begin{array}{l}\text { Título: Aumento de tejido blando } \\
\text { periimplantario con matriz de colágeno } \\
\text { porcino. Reporte de un caso. }\end{array}$ & $\begin{array}{l}\text { Título: Anatomía de la furca dentaria y } \\
\text { lesiones de furcación. }\end{array}$ \\
\hline $\begin{array}{l}\text { Objetivo. Analizar los factores relacionados con la } \\
\text { producción científica de estudiantes del pregrado. } \\
\text { Métodos. Estudio relacional, retrospectivo donde } \\
\text { participaron } 45 \text { estudiantes de la Facultad de } \\
\text { Odontología de la Universidad Nacional Mayor } \\
\text { de San Marcos que lograron publicar artículos } \\
\text { científicos desde el año } 2010 \text { hasta el } 2017 \text {. Los } \\
\text { estudiantes fueron seleccionados a partir de una } \\
\text { búsqueda manual de sus artículos en las bases } \\
\text { de datos: Scopus, PubMed, SciELO, LILACS, } \\
\text { Google Académico y Cochrane. A cada estudiante } \\
\text { se le administró un cuestionario que incluyó } \\
\text { reactivos para valorar las características personales, } \\
\text { laborales, académicas e institucionales de cada } \\
\text { estudiante. Cada factor fue relacionado con la } \\
\text { cantidad de producción científica. Resultados. Se } \\
\text { encontró una producción científica del } 10 \% \text {, el } \\
\text { pertenecer a una Sociedad Científica (OR=0,639 } \\
\text { IC95\%: } 0,43-2,32) \text {, haber participado en congresos } \\
\text { científicos (OR=0,583 IC95\%: } 0,22-1,64) \text {, haber } \\
\text { organizado eventos académicos/científicos (OR= } \\
0,58 \text { IC95\%: 0,11-2,05) y ser reconocidos por } \\
\text { sus docentes no se encontraron relacionados } \\
\text { con una mayor producción científica.(OR=0,88 } \\
\text { IC95\%:0,64-1,57), solamente hubo relación con } \\
\text { haber llevado cursos sobre redacción científica } \\
\text { (OR=0,088 IC95\%: } 0,047-0,78) \text { y mayor tiempo de } \\
\text { dedicación a la investigación (OR=0,64 IC95\%: } \\
0,12-0,71) \text {. Conclusión. La experiencia en cursos } \\
\text { sobre redacción científica se encuentra relacionada } \\
\text { con una mayor producción científica estudiantil por } \\
\text { lo que se requiere que se incentive su promoción } \\
\text { dentro del ambiente universitario. }\end{array}$ & $\begin{array}{l}\text { Una buena cantidad y grosor de tejido } \\
\text { queratinizado alrededor de implantes ha sido } \\
\text { asociado con una mejor salud periimplantaria, } \\
\text { menos pérdida ósea y una mejora en la } \\
\text { estética. El propósito de este caso clínico } \\
\text { fue evaluar un nuevo xenoinjerto de origen } \\
\text { porcino (matriz de colágeno) al ser utilizada } \\
\text { como un injerto interposicional para aumentar } \\
\text { el grosor de la mucosa periimplantaria. Son } \\
\text { pocos los casos que utilizan la matriz de } \\
\text { colágeno como sustituto del injerto conectivo } \\
\text { subepitelial alrededor de implantes. El caso } \\
\text { clínico incluyó un implante a nivel de la pieza } \\
\text { 15, en el cual durante la colocación del pilar } \\
\text { de cicatrización se aprovechó para engrosar la } \\
\text { mucosa periimplantaria utilizando una matriz } \\
\text { de colágeno. Se obtuvo un aumento de grosor } \\
\text { de } 1,5 \text { mm manteniéndose la cantidad de } \\
\text { mucosa queratinizada de } 4 \text { mm. Se concluyó } \\
\text { que la matriz de colágeno de origen porcino } \\
\text { es una buena alternativa para aumentar el } \\
\text { grosor de la mucosa periimplantaria, además } \\
\text { de reducir la morbilidad, ser de fácil manejo y } \\
\text { de fácil sutura. }\end{array}$ & $\begin{array}{l}\text { El avance de la enfermedad periodontal } \\
\text { debido principalmente a la presencia de la } \\
\text { biopelícula, provoca la pérdida de inserción } \\
\text { y de hueso a nivel de las raíces dentarias, } \\
\text { este avance de la entidad patológica } \\
\text { periodontal conlleva a nivel de los dientes } \\
\text { multiradiculares a los compromisos de } \\
\text { furcación, los cuales constituyen una lesión } \\
\text { particular y única, difícil de tratar por las } \\
\text { características anatómicas de la zona. El } \\
\text { presente artículo recopila la información } \\
\text { más actualizada y relevante de buscadores } \\
\text { como PubMed y Cochrane sobre la anatomía } \\
\text { de dientes multiradiculares, la furca } \\
\text { dentaria así como las consideraciones para } \\
\text { el diagnóstico de las lesiones de furcación. } \\
\text { La furca dentaria es la zona anatómica } \\
\text { que comprende al área de división de las } \\
\text { raíces de dientes multiradiculares; ya sean } \\
\text { premolares o molares. Las patologías que } \\
\text { acarrean a esta zona son muy complejas y } \\
\text { reciben el nombre de lesiones de furcación. } \\
\text { Se concluye que los defectos en furcaciones } \\
\text { representan un gran reto para los terapistas } \\
\text { debido a sus características anatómicas y a su } \\
\text { variabilidad en cuanto a la forma de respuesta } \\
\text { al tratamiento. La pérdida de inserción se } \\
\text { desarrolla en sentido horizontal o vertical del } \\
\text { área. Un amplio rango de modalidades de } \\
\text { tratamiento han sido dirigidos a mantener esta } \\
\text { área anatómica. }\end{array}$ \\
\hline
\end{tabular}

\section{Las palabras clave}

También denominados "descriptores" y su traducción al inglés como "keywords", son los términos más relevantes de todo el desarrollo del manuscrito. Por lo general se redactan entre 3-5 palabras clave como un segmento luego del resumen ${ }^{1}$.

De preferencia deben ser utilizadas para redactar el título. Para su localización se utilizan los tesauros (dic- cionarios de términos médicos), entre los más utilizados se encuentran los Descriptores en Ciencias la Salud (DeCS) y el "Medical Subject Headings" (MeSH o Encabezados de Temas Médicos) ${ }^{22}$. En ambos se enlistan los términos aceptados internacionalmente que permiten la identificación del manuscrito. En los DeCS se encuentran términos en español, inglés y portugués mientras que en los $\mathrm{MeSH}$ solo en inglés. 
Las funciones de las palabras clave incluyen: identificar si son del interés del lector, permitir la localización en bases de datos científicas, permitir resumir y reunir investigaciones sobre un mismo tema, al editor le permite identificar revisores del tema. Dentro de sus características se incluyen la sensibilidad y especificidad para ubicar un trabajo (debe existir un balance); cuando es muy sensible detecta trabajos similares al tema, cuando es muy específico localiza información puntual y puede perder otra información de utilidad ${ }^{3}$.

Cuando su uso es incorrecto, se corre el riesgo de no encontrar la información relevante. Su función es la de representar los conceptos principales tratados en el artículo y facilitar la recuperación en una base de datos ${ }^{3}$. En la tabla 6 se presentan ejemplos de palabras clave acorde a los temas de investigación.

Tabla 6. Ejemplos de palabras clave acorde a los tipos de artículo científico

\begin{tabular}{|c|c|c|}
\hline Artículo original & Reporte de caso & Artículo de revisión \\
\hline $\begin{array}{l}\text { Título: Factores que contribuyen en la } \\
\text { producción científica estudiantil. El caso de } \\
\text { Odontología. }\end{array}$ & $\begin{array}{l}\text { Título: Aumento de tejido blando } \\
\text { periimplantario con matriz de colágeno porcino. } \\
\text { Reporte de caso. }\end{array}$ & $\begin{array}{l}\text { Título: Anatomía de la furca dentaria y lesiones } \\
\text { de furcación. }\end{array}$ \\
\hline $\begin{array}{l}\text { Palabras clave: Bibliometría; Comunicación } \\
\text { y divulgación científica; Odontología; Perú; } \\
\text { Publicaciones de divulgación científica. }\end{array}$ & $\begin{array}{l}\text { Palabras clave: Colágeno; Estética; Implantes } \\
\text { dentales. }\end{array}$ & $\begin{array}{l}\text { Palabras clave: Defectos de furcación; } \\
\text { Enfermedades periodontales; Pérdida de la } \\
\text { inserción periodontal; Periodontitis; Raíz del } \\
\text { diente. }\end{array}$ \\
\hline $\begin{array}{l}\text { Keywords: Bibliometrics; Dentistry; Peru; } \\
\text { Publications for science diffusion; Scientific } \\
\text { communication and diffusion. }\end{array}$ & $\begin{array}{l}\text { Keywords: Collagen; Dental implants; } \\
\text { Esthetics. }\end{array}$ & $\begin{array}{l}\text { Keywords: Furcation defects; Periodontal } \\
\text { diseases; Periodontal attachment loss; } \\
\text { Periodontitis; Tooth root. }\end{array}$ \\
\hline
\end{tabular}

\section{Conclusiones}

El título de un artículo científico es el segmento que sintetiza la idea central de la investigación, su redacción debe ser cautelosa de tal forma que describa el contenido de forma clara, exacta y precisa; el investigador debe preguntarse cómo quisiera que su investigación sea localizada en una base de datos. Del mismo modo los componentes de un resumen deben ser suficientes para que el lector comprenda el contenido básico de la investigación, en muchas ocasiones será el único segmento que lea, motivo por el cual su redacción debe ser minuciosa aportando información sustantiva. Es importante considerar las guías sobre elaboración de resúmenes para cada tipo de estudio; así como los requerimientos que algunas revistas sugieren durante el envío del manuscrito. Considerar que una buena relación entre el título, resumen y palabras clave facilita la ubicación en los sistemas de información y bases de datos además de ser agradable para el lector y editores.

\section{Referencias bibliográficas}

1. Sánchez UA. Manual de Redacción Académica e Investigativa: Cómo escribir, evaluar y publicar artículos. En: Sánchez UA. Estructuras textuales, artículos de investigación, caracterizaciones y ponencias. Medellín: Católica del Norte Fundación Universitaria; 2011. p. 147-50.

2. Collado VS. Redacción científica: Algunos errores frecuentes. Biociencias. 2006; 4: 3-8.

3. Aranda TE, Mitru TN, Costa AR. ABC de la redacción y publicación médico-científica. En: Mitru TN. El título del artículo. 2da ed. Madrid: Cooperación Cultural Exterior; 2009. p. 61-70.

4. Letchford A, Moat HS, Preis T. The advantage of short paper titles. R Soc Open Sci. 2015;2(8):150266. doi: $10.1098 /$ rsos. 150266 .

5. Day RA. Cómo escribir y publicar trabajos científicos. M. Sáenz. (Trad.). En: Day RA. Cómo preparar el título. 3era ed. Washington: Organización Panamericana de la Salud-OPS; 2005. p. 15-7.

6. American Psychological Association-APA. Manual de publicaciones. Miroslava Guerra Frías. (Trad.) 3ra ed. México D.F: Manual Moderno; 2010.

7. Simera I, Altman DG. Reporting medical research. Int J Clin Pract. 2013; 67(8): 710-6. doi: 10.1111/ ijcp. 12168.

8. Moher D, Hopewell S, Schulz KF, Montori V, Gøtzsche PC, Devereaux PJ et al. CONSORT 2010 explanation and elaboration: updated guidelines for reporting parallel group randomised trial. BMJ. 2010; 340:c869. doi: https://doi.org/10.1136/bmj.c869.

9. Welch V, Petticrew M, Tugwell P, Moher D, O'Neill J et al. PRISMA-Equity 2012 Extension: Reporting Guidelines for Systematic Reviews with a Focus on Health Equity. PLoS Med. 2012; 9(10): e1001333. doi:10.1371/ journal.pmed.1001333.

10. Vandenbroucke JP, Von Elm E, Altman DG, Gøtzsche PC, Mulrow C, Pocock SJ et al. Mejorar la comunicación de estudios observacionales en epidemiología (STROBE): explicación y elaboración. Gac Sanit. 2009; 23(2):158e1-158e28. http://dx.doi.org/10.1590/S021391112009000200015 .

11. Cohen JF, Korevaar DA, Gatsonis CA, Glasziou PP, Hooft L, Moher D et al. STARD for Abstracts: essential items for reporting diagnostic accuracy studies in journal or conference abstracts. BMJ. 2017;358:j3751. doi: 10.1136/bmj.j3751.

12. Chan AW, Tetzlaff JM, Altman DG, Laupacis A, Gøtzsche PC, Krleža-Jeric K et al. SPIRIT 2013 statement: defining standard protocol items for clinical trials. Ann Intern Med. 2013; 158:200-7.

13. Gagnier JJ, Riley D, Altman DG, Moher D, Sox H, Kienle G, CARE Group. The CARE guidelines: consensus-based clinical case reporting guideline development. Dtsch Arztebl Int. 2013;110(37):603-8. doi: 10.3238/ arztebl.2013.0603. 
14. Ferriols R, Ferriols F. Escribir y publicar un artículo científico original.2da ed. Barcelona: Ediciones Mayo; 2005.

15. Winck JC, Fonseca JA, Azevedo LF, Wedzicha JA. To publish or perish: how to review a manuscript. Rev Port Pneumol. 2011;17(2):96-103.

16. International Committee of Medical Journal Editors. Recommendations for the conduct, reporting, editing, and publication of scholarly work in medical journals (updated december 2017). [Consultado el 28 de enero 2018]. Accesible en: http://www.icmje.org/recommendations/.

17. Driggers RG. How Do You Write a Great Abstract and Why Is It Important? Opt Eng. 2010;49(6):060101-1. doi:10.1117/1.3446796.

18. Andrade C. How to write a good abstract for a scientific paper or conference presentation. Indian J Psychiatry. 2011;53(2):172-5.
19. Mott S. The process of writing an abstract. J Pediatr Nurs. 2014;29(4):383-5.

20. Beller EM, Glasziou PP, Altman DG, Hopewell S, Bastian H, Chalmers I, et al. PRISMA for Abstracts: reporting systematic reviews in journal and conference abstracts. Plos Med. 2013;10(4):e1001419. doi: 10.1371/ journal.pmed.1001419.

21. Boutron I, Altman DG, Moher D, Schulz KF, Ravaud P; CONSORT NPT Group. CONSORT Statement for Randomized Trials of Nonpharmacologic Treatments: A 2017 Update and a CONSORT Extension for Nonpharmacologic Trial Abstracts. Ann Intern Med. 2017;167(1):40-47. doi: 10.7326/M17-0046.

22. Comité Internacional de Editores de Revistas Médicas. Requisitos de Uniformidad para los manuscritos enviados a revistas biomédicas: escritura y proceso editorial para la publicación de trabajos biomédicos. Rev Esp Cardiol. 2004; 57(6):538-56. 